\title{
Patient Perspectives of Sharing Experiences in Group-Based Diabetes Education: A Second-Order Analysis
}

\author{
Vibeke Stenov, Ingrid Willaing \\ Steno Diabetes Center, Health Promotion Research, Gentofte, Denmark \\ Email:vbse@steno.dk
}

Received 25 June 2015; accepted 5 January 2016; published 8 January 2016

Copyright (C) 2016 by authors and Scientific Research Publishing Inc.

This work is licensed under the Creative Commons Attribution International License (CC BY).

http://creativecommons.org/licenses/by/4.0/

(c) (i) Open Access

\begin{abstract}
The study aimed to develop a second-order theoretical analysis based on qualitative interviews exploring the experiences of persons with diabetes attending group-based diabetes education. Bruner's cultural psychology and White and Epston's narrative therapy provided a theoretical foundation for the analysis. The analysis indicated that telling and listening to stories from everyday life with diabetes in a peer group can assist participants in reinterpreting ways of living with diabetes. Sharing experiences with peers in group-based diabetes education seems to be supportive and useful when individuals are trying to find strategies to cope with diabetes in everyday life.
\end{abstract}

\section{Keywords}

Diabetes, Health Education, Narratives, Peer Support, Qualitative Methods

\section{Introduction}

Aging populations and changes in lifestyle have led to an increasing prevalence of diabetes. Patient education has been identified as an important method for improving health in people with diabetes through enhancement of disease self-management skills (Hansen et al., 2014). There is ongoing academic discussion about effective methods for diabetes education. Research has generally focused on biomedical outcomes such as glycosylated haemoglobin (HbA1c), lipids, weight loss and medication rather than psychosocial experiences of living with diabetes (Cooper et al., 2003; Haakanson et al., 2012). Measurement of biomedical outcomes is valuable because rigorous glucose control can greatly reduce the incidence of serious complications in patients with diabetes (Costello, 2013). Nevertheless, these measures provide limited information about the necessary ingredients for improving self-management of diabetes.

How to cite this paper: Stenov, V., \& Willaing, I. (2016). Patient Perspectives of Sharing Experiences in Group-Based Diabetes Education: A Second-Order Analysis. Psychology, 7, 12-18. http://dx.doi.org/10.4236/psych.2016.71003 
Self-care actions in everyday life with diabetes also need to be understood in terms of the psychosocial aspects of living with a chronic illness (Haas et al., 2013; Harrison et al., 2011; Ockleford et al., 2008). Living with a chronic illness such as diabetes often implies limitations because of difficulties in finding strategies to manage illness in everyday life. People with diabetes often experience psychosocial problems and challenges that reduce quality of life (Peyrot et al., 1999; Peyrot et al., 2013). Depression and anxiety, which have been reported to be twice as prevalent among people with diabetes as in the general population, have been associated with poor metabolic control and increased risk of late complications (DeVries et al., 2004; Grigsby et al., 2002; Lustman, 2000).

In recent years, patient education has undergone considerable changes, such as increased interest in how healthcare professionals can facilitate motivation for change among persons with chronic disease, e.g., motivational interviewing, active listening and empowerment. These perspectives often have an individualistic approach and build on the relationship between the individual patient and the health care professional. However, sharing experiences in group-based patient education may reduce the burden of living with diabetes and lead to improved outcomes (Harrison et al., 2011; Due-Christensen et al., 2012). The foundation of group-based patient education is social learning techniques, which include the opportunity to meet with peers who are diagnosed with diabetes and share experiences in a reassuring and supportive environment. This may lead to a specific discussion of common issues within the framework of living with diabetes (Barlow et al., 2005). The exchange of experiences is known to promote effective learning; knowledge acquisition and conceptual growth arises from the sharing of perspectives and testing of ideas with others (Haakanson et al., 2011). Furthermore, groupbased patient education allows efficient and cost-effective delivery of patient education. Previous research shows that group processes are often the essential element in the success of education programs; participants may perceive them as more important than biomedical content, e.g., disease-specific topics addressed by health care professionals (Harrison et al., 2011; Ockleford et al., 2008; Nossum, 2013).

The aim of the present paper is to develop an empirical framework for peer-to-peer sharing of experiences among participants in group-based diabetes education, using qualitative interview data and second-order theoretical analysis to generate a meaningful explanation of patient needs and preferences. The theory is based on the application of cultural psychology to education developed by Jerome Bruner. Additionally, perspectives from narrative therapy developed by Michael White and David Epston, drawing on Bruner's initial concepts (White \& Epston, 1989), are used in the-second order analysis. Bruner's psycho-cultural approach to education represents an intersection between the individual mind and the influence of culture in the process of constructing identity. This narrative-based approach looks for meaning as to how sharing experiences with peers in groupbased diabetes education can support well-being and self-management.

\section{Method}

The group-based diabetes education program discussed here was established in 1997 in a Danish university hospital. The program was based on the idea that confluent pedagogy can facilitate the process of transforming new knowledge into useful strategies for self-management in everyday life with diabetes. Confluent pedagogy integrates disease-specific knowledge provided by health professionals, experiences of life with diabetes among persons with diabetes and a practical learning element, such as a trip to the local supermarket to buy groceries. The program was provided to groups of 10 - 12 adult participants consisting of patients with diabetes of both genders and a varying number of their relatives. The education program comprised four days with seven hours of educational sessions per day, a total of 13 sessions. Four health professionals were involved in each program: diabetologists, nurses, dieticians and podiatrists.

\subsection{Participants}

Immediately after the final day of three programs, we conducted focus groups that consisted of four to six persons with diabetes. Participant perceptions of the program were subsequently explored through 19 semi-structured individual telephone interviews conducted within three months of the end of the program. Telephone interviews, rather than face-to-face conversations, were necessary due to the geographical dispersion of participants.

Sampling criteria included varying age, gender and diabetes characteristics such as diabetes type and treatment (Table 1). Persons with diabetes were asked about their general experience and assessment of the entire 
diabetes education program, as well as specific experiences and assessments of each session. Data collection took place from April 2012 to December 2012.

\subsection{Analysis}

A second-order theoretical analysis was used to construct a theoretical explanation of participant experiences, based on the application of Bruner's theory of cultural psychology and White and Epston's theory of narrative therapy to data gathered from the focus groups and interviews. This method includes two steps of theoretical analysis: first-order analysis and second-order analysis.

First-order analysis. First-order theoretical analysis is the process of constructing a theoretical explanation by directly translating descriptive categories into theoretical categories (Shkedi, 2004). The first-order theoretical analysis translates descriptive categories into theories that are grounded in the narratives, perceptions and assessments of people with diabetes sharing experiences with other persons with diabetes.

The descriptive categories were generated using systematic text condensation (Malterud, 2012). Systematic text condensation consists of four steps: a) reading all the material to obtain an overall impression and bracketing previous preconceptions; b) identifying units of meaning representing different aspects of participants' perceptions of the educational program; c) condensing and abstracting the meaning within each coded group; and d) summarizing the contents of each coded group to generalize descriptions and concepts reflecting the most important experience from the voices of the persons with diabetes (Malterud, 2012). To avoid repetition, results of both focus group and follow-up interviews are presented together.

Second-order analysis. After the first-order analysis was complete, the authors noted that some theoretical patterns could be discerned and decided to continue the study through second-order theoretical categorization. In second-order categorization, analysis was based on the first-order categories, which were used to arrive at a new level of understanding and a more refined theoretical categorization (Shkedi, 2004). In this step, patient experiences of attending group-based diabetes education were explained through a narrative-based framework, which was used as a model for constructing a theoretical explanation of the effectiveness of peer-to-peer sharing. This theoretical approach enabled meaningful description of the importance of sharing experiences in group-based patient education.

\section{Results}

The first-order analysis revealed two preliminary descriptive categories that emerged from interviews: 1) learning from each other is the essential element of the patient education program; and 2) people with diabetes experience identical challenges in everyday life with diabetes. Figure 1 demonstrates the relationships between empirical categories from the first-order analysis and categories in the second-order theoretical categorization.

The core theoretical categories define the phenomenon and the descriptive categories represent the characteristics of the theoretical explanation. We developed two theoretical categories in the second-order interpretative synthesis of themes: 1) interactive learning enables construction of new narratives; and 2) the collective structures influence individual narratives.

Table 1. Sample and participant characteristics. Characteristics of 19 interviewed participants.

\begin{tabular}{ccc}
\hline & Interview participants & Focus group participants \\
& $\mathrm{N}=19$ & $\mathbf{N}=16$ \\
\hline Age, years, range & $28-72$ & 15 \\
Female, no. & 13 & \\
Diagnosis, no. & & 9 \\
Type 1 diabetes & 10 & 7 \\
Type 2 diabetes & 9 & 4 \\
Marital status, no. & & 12 \\
Single/divorced/widowed & 3 & \\
Married/with a partner & 16 & \\
\hline
\end{tabular}




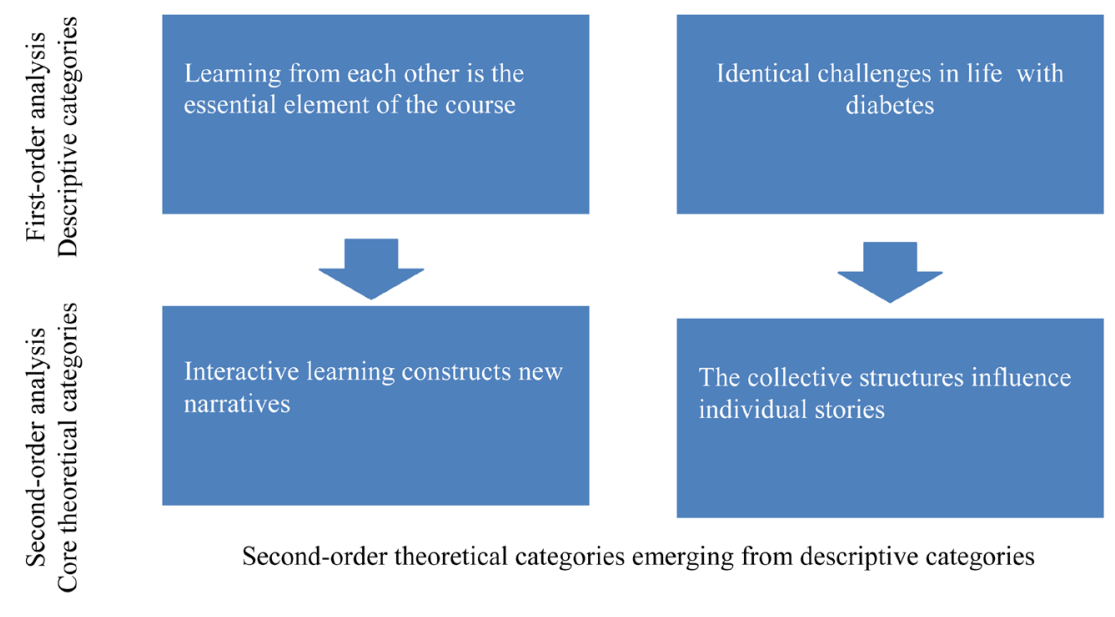

Figure 1. Second-order theoretical categories emerging from descriptive categories.

\subsection{Interactive Learning Constructs New Narratives}

According to Bruner, learning happens primarily through interacting with other participants, not by listening to teachers without involving participants (Bruner, 1998). The room where education takes place should be a sub-community of mutual learners, with the educator orchestrating the process to encourage the sharing of experiences (Bruner, 1998). Bruner's view of the educator role and how learning takes place through participant interaction was reflected in the empirical findings.

In interviews, when we asked about their general experience of the course, participants immediately referred to sharing experiences from everyday life with peers as the essential element of the education program. Several individuals with diabetes found it more valuable to share experiences with peers when trying to find new strategies in everyday life with diabetes than to participate in scheduled sessions where educators provided general information about diabetes. One participant stated clearly that sharing experiences was the most useful element of the course, and another said it was most useful "to be with other people who had diabetes."

Participants reported that educators did not have the same understanding of what was meaningful in everyday life with diabetes as did their peers participating in the course. Individuals with diabetes expressed a substantial need for talking to someone who "really" understood them. One participant stated it this way:

"I do not know any nurses who have diabetes, I do not know any doctors who have it, so they do not know how I feel."

Even participants who had initially felt uncomfortable about being in a group reported that being with peers provided comfort and strength:

"I really need to talk to someone with the same illness. Before I came here I didn't think it was necessary, but it was very good for me and I felt very good afterwards."

Perspectives from Bruner and narrative therapy can help explain why sharing of experiences can potentially enhance well-being and self-management. Bruner emphasizes the role of language in building narratives that construct identity throughout life. Group-based diabetes education is a natural setting for telling and listening to stories from everyday life with diabetes and integrating these stories into one's own life with diabetes, maintaining or reconstructing a positive identity to achieve increased well-being. To reconstruct a positive identity with diabetes and generate new perspectives, reflexivity is fundamental [NOTE: A definition of reflexivity would be helpful, and it seems like a citation is needed to support this assertion]. Reflexivity is also the key to expanding one's own story by considering past experiences to change present perceptions or changing past perceptions based on present experiences. Past experiences can be reframed in different and more constructive ways through reflexivity. In the empirical data, it was very clear that peer-to-peer sharing was very useful for gaining insights into other ways of coping with diabetes-related problems. This provided reflexivity that engendered new strategies, generated inspiration to manage the condition, and helped participants discover useful strategies to cope with and manage diabetes in everyday life. As one person stated: 
"When you are in a similar situation then you can say: "Oh the others [participants] did it this way and it felt like that."

\subsection{The Influence of the Collective Structures}

According to Bruner, narratives are not only generated by the individual but are also provided through the cultural setting (Bruner, 1996). This means that collective and social structures influence individual ways of living with diabetes. This theoretical approach creates the possibility of recognizing that everyday issues with diabetes are not individually caused but are, rather, collectively caused. This means that participants in group-based patient education had different stories to tell about living with diabetes, but every story had some elements that were identical to those of narratives told by most of the participants. These identical stories can create a feeling that "things went the same way for me as they did for others in the same situation", conferring a feeling of normality and a decreased sense of isolation, reducing loneliness and emotional burden. The importance of searching for identical factors in everyday life with diabetes was described by one participant:

"I am type one with type one problems, that is why I need to be together with type one persons (...) it is important sometimes to spend time with peers."

The influence of collective and social structures on individual ways of living with diabetes was identified several times in the empirical data; the majority of participants with diabetes expressed that the benefit of participating in the group was the feeling of not being alone. As one participant expressed it, "it is nice not to be the only one who has been in this situation.”

Participants with diabetes expressed feeling relief when they learned that other people had similar thoughts, fears, feelings and challenges in everyday life. Among the themes participants with diabetes considered as challenging and very valuable issues to discuss with peers were the fear and dread of needle pricks when injecting insulin. Another important aspect to discuss was personal experiences with low blood glucose and the fear of future episodes of hypoglycaemia. Additionally, the issue of how to engage their social network in their illness was experienced by some participants as particularly difficult and how peers coped with this was an important issue.

When persons with diabetes realize that problems possess collective traits rather than being exclusively caused by individuals, the theoretical explanation can incorporate the concept of externalization. According to Bruner, externalization makes it more possible to embody thoughts and intentions in a form where the person and the specific challenge with diabetes are separate entities (Bruner, 1998). The problem is no longer within the person with diabetes. When individuals with diabetes externalize challenges, they gain the opportunity to reflect as peers who have been in similar situations. This can result in new ways of living with diabetes and the possibility of enhancing positive changes to handle challenges. This was stated by one participant:

"Especially the part where we share some of the experiences we've had, I think it was very positive, (...) how the others experience everyday life and the small things and annoying things, so in that way I definitely think, that to learn from each other's experiences is really important."

All persons with diabetes emphasized the importance of being in a supportive group environment and almost all expressed a need for continued contact with each other after the end of the program They were unsure of their ability to maintain motivation for goal setting when they were no longer in a supportive environment. Participants with diabetes assumed that continuous feedback from the rest of the group after the end of the program would provide motivation for continued action and inspiration:

"I would like after six months or a year, to make a follow-up for the entire group and then hear how everybody is doing? (...) What have you learned and what have you used and not used?"

\section{Discussion}

By using a narrative-based approach to establish a theoretical foundation, we have shown that telling peer-topeer sharing can create alternative stories which help participants with diabetes identify new solutions through an atmosphere of mutual support (Marrero, 2013). Individuals with diabetes found it more valuable to exchange experiences with peers than to participate in scheduled sessions provided by health care professionals. This may encourage health care professionals to facilitate narrative-based techniques in diabetes education, which requires 
that they actively listen to individuals with diabetes and integrate their needs into the curriculum. However, the evidence suggests that it is often challenging for health care professionals to encourage and facilitate a participant-directed discussion in practice. Health care professionals are typically socialized into a biomedical paradigm (derived from the treatment of acute illness), in which they act as experts who feel responsible for solving their patients' problems. Health care professionals often expect patients to follow their recommendations (Adolfsson, 2004; Anderson, 2005).

A fundamental issue is the asymmetry of power between patients and health care professionals. Change requires a more equitable distribution of power between individuals with diabetes and health care professionals (Anderson, 1996). Facilitation is considered a useful tool to increase peer support in patient education and empower participants and is supported by principles of adult education, which suggest that it is more likely that significant learning takes place when persons with diabetes find a subject relevant to their personal situations. Facilitation requires a dialogue with individuals with diabetes (Cooper et al., 2003; Haas et al., 2013). However, the ability of health care professionals to act as facilitators is not the only important factor when trying to obtain successful outcomes from group-based patient education. Several other factors, such as the selection of the right mixture of patients in the group, may also be essential for successful outcomes.

In this study, participants with diabetes expressed the perception that living with diabetes often created many questions and few clear answers. A wide variety of themes and topics related to experiences and feelings in everyday life with diabetes were considered important and meaningful to share and discuss during group education. The educator may open the possibility of addressing the psychosocial aspect of patient education, which can interfere with diabetes management when unaddressed and is an essential component of achieving glycamic goals and improving long-term outcomes (Peyrot et al., 2005).

Strengths of the study include the use of data collected systematically in four complete patient education programs with persons with diabetes of varying age and both genders and types of diabetes. The sample size and amount of empirical data are relatively large. Limitations include the fact that all interviews were conducted in a single hospital setting, potentially limiting the generalizability of our findings to other settings. However, the diabetes education program described here is the largest and most comprehensive in Denmark, involving many educators. In addition, all interviews were conducted by telephone, a process that has inherent limitations for establishing rapport and observing nonverbal signals.

This study indicates that peer-to-peer sharing of experiences can assist with reinterpreting ways of coping and living with diabetes. However, the health care professionals conducting the diabetes education program had no training in narrative therapy; the outcomes reported here require further investigation. Further research is also needed into the effect of training health care professionals in using narrative and facilitative approaches in diabetes education. Finally, the inclusion of systematic peer support in diabetes education should be further investigated.

\section{Practical Implications}

When planning diabetes education, it is pivotal that educators be aware of the importance of patients with diabetes sharing stories with one another and make opportunities for peer-to-peer sharing an integral part of the educational intervention. To succeed, however, health care professionals involved in patient education must be able to both facilitate storytelling and catalyse reflections among peers to construct new narratives. Additionally, including the right mixture of patients in the group, i.e., those with a range of personalities and problems, is also an important aspect of effective group-based patient education.

\section{Acknowledgements}

Nete Schwennesen was the principal investigator for a larger study in which the present study was nested and as such managed the data collection. We thank all persons with diabetes and health professionals at the Department of Endocrinology, Odense University Hospital for their participation and support throughout the study.

\section{References}

Adolfsson, E. T. et al. (2004). Implementing Empowerment Group Education in Diabetes. Patient Education and Counseling, 53, 319-324. http://dx.doi.org/10.1016/j.pec.2003.07.009 
Anderson, R. M., \& Funnell, M. M. (2005). Patient Empowerment: Reflections on the Challenge of Fostering the Adoption of a New Paradigm. Patient Education and Counseling, 57, 153-157. http://dx.doi.org/10.1016/j.pec.2004.05.008

Barlow, J. H., Bancroft, G. V., \& Turner, A. P. (2005). Self-Management Training for People with Chronic Disease: A Shared Learning Experience. Journal of Health Psychology, 10, 863-872. http://dx.doi.org/10.1177/1359105305057320

Costello, J. F. (2013). Roles and Strategies of Diabetes Support Group Facilitators: An Exploratory Study. Diabetes Educator, 39, 178-186. http://dx.doi.org/10.1177/0145721713476347

Cooper, H.C., Booth, K., \& Gill, G. (2003). Patients’ Perspectives on Diabetes Health Care Education. Health Education Research, 18, 191-206. http://dx.doi.org/10.1093/her/18.2.191

DeVries, J. H., Snoek, F. J., \& Heine, R. J. (2004). Persistent Poor Glycaemic Control in Adult Type 1 Diabetes. A Closer Look at the Problem. Diabetic Medicine, 21, 1263-1268. http://dx.doi.org/10.1111/j.1464-5491.2004.01386.x

Due-Christensen et al. (2012). Can Sharing Experiences in Groups Reduce the Burden of Living with Diabetes, Regardless of Glycaemic Control? Diabetic Medicine, 29, 251-256. http://dx.doi.org/10.1111/j.1464-5491.2011.03521.x

Grigsby, A. B. et al. (2002). Prevalence of Anxiety in Adults with Diabetes a Systematic Review. Journal of Psychosomatic Research, 53, 1053-1060. http://dx.doi.org/10.1016/S0022-3999(02)00417-8

Haankanson, C. et al. (2012). Learning about Oneself through Others: Experiences of a Group-Based Patient Education Programme about Irritable Bowel Syndrome. Scandinavian Journal of Caring Sciences, 26, 738-746. http://dx.doi.org/10.1111/j.1471-6712.2012.00990.x

Haakanson, C. et al. (2011). Learning to Live with Irritabel Bowel Syndrome: The Influence of a Group-Based Patient Education Programme on Peoples’ Ability to Manage Illness in Everyday Life. Scandinavian Journal of Caring Sciences, 25, 491-498. http://dx.doi.org/10.1111/j.1471-6712.2010.00855.x

Haas, L., Maryniuk, M., Beck, J. et al. (2013). National Standards for Diabetes Self-Management Education and Support. Diabetes Care, 36, 100-108. http://dx.doi.org/10.2337/dc13-S100

Hansen, U. M., Engelund, G., Rogvi, S., \& Willaing, I. (2014). The Balancing Person: An Innovative Approach to PersonCentered Education in Chronic Illness. European Journal for Person Centered Healthcare, 2, 290-302.

Harrison, M., Fullwood, C., Bower, P. et al. (2011). Exploring the Mechanisms of Change in the Chronic Disease Self-Management Programme: Secondary Analysis of Data from a Randomised Controlled Trial. Patient Education and Counseling, 85, 39-47. http://dx.doi.org/10.1016/j.pec.2010.10.026

Lustman, P. J., Anderson, R. J., Freedland, K. E. et al. (2000). Depression and Poor Glycemic Control: A Meta-Analytic Review of the Literature. Diabetes Care, 23, 934-942. http://dx.doi.org/10.2337/diacare.23.7.934

Malterud, K. (2012). Systematic Text Condensation: A Strategy for Qualitative Analysis. Scandinavian Journal of Public Health, 40, 795-805. http://dx.doi.org/10.1177/1403494812465030

Marrero, D. G. (2013). Twenty-First Century Behavioral Medicine: A Context for Empowering Clinicians and Patients with Diabetes: A Consensus Report. Diabetes Care, 36, 463-470. http://dx.doi.org/10.2337/dc12-2305

Nossum, R., Rise, M. B., \& Steinsbekk, A. (2013). Patient Education-Which Parts of the Content Predict Impact on Coping Skills? Scandinavian Journal of Public Health, 41, 429-435. http://dx.doi.org/10.1177/1403494813480279

Ockleford, E., Shaw, R. L., Willars, J., \& Dixon-Woods, M. (2008). Education and Self-Management for People Newly Diagnosed with Type 2 Diabetes: A Qualitative Study of Patients’ Views. Chronic Illness, 4, 28-37. http://dx.doi.org/10.1177/1742395307086673

Peyrot, M., McMurry, J., \& Kruger, D. F. (1999). A Biopsychosocial Model of Glycemic Control in Diabetes: Stress, Coping and Regimen Adherence. Journal of Health and Social Behavior, 40, 141-158. http://dx.doi.org/10.2307/2676370

Peyrot, M., Rubin, R. R. et al. (2005). Psychosocial Problems and Barriers to Improved Diabetes Management: Results of the Cross-National Diabetes Attitudes, Wishes and Needs (DAWN) Study. Diabetic Medicine, 22, 1379-1385.

http://dx.doi.org/10.1111/j.1464-5491.2005.01644.x

Peyrot, M., Burns, K. K., Davies, M. et al. (2013). Diabetes Attitudes Wishes and Needs 2 (DAWN2): A Multinational, Multi-Stakeholder Study of Psychosocial Issues in Diabetes and Person-Centred Diabetes Care. Diabetes Research and Clinical Practice, 99, 174-184. http://dx.doi.org/10.1016/j.diabres.2012.11.016

Shkedi, A. (2004). Second Order Theoretical Analysis: A Method for Constructing Theoretical Explanation. International Journal of Qualitative Studies in Education, 17, 627-646. http://dx.doi.org/10.1080/0951839042000253630 